ERIK CIRAVEGNA

ESCUELA DE DISEÑO

FACULTAD DE ARQUITECTURA,

DISEÑO Y ESTUDIOS URBANOS

PONTIFICIA UNIVERSIDAD

CATÓLICA DE CHILE

SANTIAGO, CHILE

ERIK.CIRAVEGNA@UC.CL

Este ensayo surge a partir del trabajo doctoral del autor, sistematizando y complementando parte de sus resultados. La investigación de doctorado se desarrolló entre los años 2005 y 2008 en el Departamento de Diseño del Politecnico di Milano. Tesis titulada Progettare la qualità comunicativo-informativa dell'imballaggio. Metodi e strumenti per l'accesso ai contenuti informativi nel packaging design [Diseñar la calidad comunicativa de la información en el packaging. Métodos y herramientas para el acceso a los contenidos informativos en el proyecto de envases]". Profesora guía: Prof. V. L. Bucchetti; tutor científico: Prof. G. Baule; coordinador del programa de doctorado: Prof. E. Manzini. La investigación doctoral fue financiada por una beca del Ministerio de la Universidad y la Investigación del Gobierno de Italia.

Cómo citar: Ciravegna, E. (2017). Diseño de packaging. Una aproximación sistémica a un artefacto complejo. RChD: creación y pensamiento, 2(3), 1-17. DOI: $10.5354 / 0719-837 \times .2017 .47825$

Revista Chilena de Diseño,

RChD: creación y pensamiento

Universidad de Chile

2017, 2(3)

http://rchd.uchile.cl

\section{Diseño de packaging. Una aproximación sistémica a un artefacto complejo}

\author{
Packaging Design. A Systemic Approach to a Complex Artifact
}

Resumen. El packaging es un artefacto complejo, que con el tiempo ha ampliado considerablemente sus funciones. Es un objeto de uso (prótesis instrumental), que permite contener un producto, protegerlo, almacenarlo, transportarlo, y al mismo tiempo, favorecer su interacción física con el usuario. Además es un dispositivo de comunicación (prótesis comunicativa), caracterizado por funciones de tipo apelativo, persuasivo, informativo, prescriptivo, entre otros.

Por su naturaleza multifacética, el proyecto de un envase requiere diferentes habilidades y la intervención de múltiples disciplinas: un packaging, por lo tanto, debe entenderse como el resultado de un conjunto integrado de elecciones realizadas por una pluralidad de actores que desempeñan, cada uno, un rol específico - directo o indirecto- en su definición. Dichos actores, junto con las relaciones que entre ellos se establecen a lo largo del ciclo de vida del producto, conforman el llamado "sistema-packaging".

Dentro de este sistema, el Diseño -y específicamente el Diseño de comunicación- juega un papel relevante de dirección y mediación, capaz de dar forma a través de la síntesis proyectual a soluciones que sean expresión de la confluencia de las necesidades de las distintas partes implicadas y las múltiples funciones del artefacto, conectando la dimensión comunicativa a la dimensión más estrictamente prestacional y operativa.

Palabras clave: diseño de envases, dispositivo de comunicación, objeto de uso, packaging, sistematización.

\begin{abstract}
Packaging is a complex artifact, which has considerably expanded its functions over time. It is an object of use (instrumental prosthesis), which allows to contain the product, protect it, store it, transport it, and at the same time, facilitate its physical interaction with the user. Also, it is a communication device (communication prosthesis), characterized by functions, among others, of appellative, persuasive, informative and prescriptive kind. For its multifaceted nature, the project of a package requires different skills and the intervention of multiple disciplines: for that reason, it should be understood as the result of an integrated set of choices made by a plurality of actors, where each performs a specific role - direct or indirect - in its definition. These actors, together with the relationships that are established throughout the product life cycle, define the so-called "packaging-system". Within this system, Design - and specifically Communication Design - may play an important role in direction and mediation, potentially giving shape through the project synthesis to solutions which are an expression of the confluence of the needs of the different parties involved and the multiple functions of the artifact, connecting the communicative dimension to a strictly instrumental dimension.
\end{abstract}

Keywords: communication device, object of use, packaging, packaging design, systematization. 
1. El inmenso universo de los objetos que nos rodean, incluido el envase, es un sistema que circunscribe y regula las conductas y las ideologías de una sociedad de consumo (Baudrillard, 1968).

Sobre la naturaleza "híper-artificial" del ambiente semiótico y sensorial en el que vivimos, ver también el texto de Ezio Manzini (1990).

2. El término "significación" es un concepto semiótico y se refiere a la propiedad que tiene cada cosa (por ejemplo, un objeto, un fenómeno natural, una persona, entre otros), como "signo" (significante), para referirse a un "contenido" (significado). Ver, por ejemplo, el trabajo de Eco (1973; 1975b) y de Marsciani y Zinna (1991).

3. Sobre el concepto de "prótesis", ver las reflexiones de Eco (1975a; 1997), Anceschi (1988, 1992a), Maldonado (1992).

\section{Introducción: packaging y cotidianidad contemporánea}

Entre los objetos que pueblan nuestra vida cotidiana, el packaging es sin duda uno de aquellos que más capilarmente están presentes en nuestros entornos: en el hogar, el trabajo, el tiempo libre, durante un viaje, entre otros. No es solamente un objeto material que acompaña a la casi totalidad de los productos que se compran y consumen, sino también el protagonista -más o menos explícito- de situaciones mediales que se presentan en el diario vivir, como la televisión o la publicidad en sus diversas formas, donde el envase asume un rol de "actor massmediático" (Bucchetti, 1999, pp. 54-57). Por estas razones, al igual que otros objetos cotidianos, su amplia difusión contribuye a modificar el espacio social en el que se inserta y determina su estética cotidiana. ${ }^{1}$ Además de ser un objeto de uso con funciones de contención, protección, transporte, preservación, entre otros., el envase es también un "objeto de sentido", es decir, creador e instaurador de significación. ${ }^{2}$ A través de sus propiedades sensoriales y semánticas -expresadas no solamente por la configuración de los elementos estrictamente gráficos, sino del conjunto de los componentes verbales, icónicos, morfológicos, estructurales, materiales y de superficie- activa la producción de sentido del producto y favorece la relación de este con su destinatario (usuario/consumidor).

A lo largo del siglo pasado, gracias principalmente a las innovaciones tecnológicas en el ámbito productivo y logístico (los resultados de la investigación en el campo de los materiales inteligentes, las nanotecnologías, los procesos automatizados de producción y envasado, entre otros), los envases han desarrollado y fortalecido sus funciones de uso. Sin embargo, lo que más ha marcado su evolución en las últimas décadas ha sido la expansión de sus funciones comunicativas, al punto que el packaging es considerado, en el actual panorama del consumo, como uno de los elementos más importantes en los procesos de construcción y fortalecimiento de la identidad del producto, el centro de gravedad de su "instrumentario comunicativo" (Bucchetti, 1999, p. 50). Desde la perspectiva del Diseño, tanto el proyecto de una nueva solución de packaging como la mejora de un envase existente, suponen saber enfrentar la complejidad y naturaleza multifacética de este artefacto: por una parte, adquiriendo las diferentes habilidades que su proyecto implican; por otra, aprendiendo a gestionar las relaciones que se establecen con los múltiples ámbitos disciplinares involucrados en su desarrollo y producción. Por esta razón, se estima relevante proponer, para el área del diseño de packaging, la sistematización de un marco conceptual, basado en la revisión de la literatura y en algunas reflexiones sobre las funciones del envase y el rol del diseñador en relación con su proyecto.

\section{Un artefacto complejo: packaging como objeto de uso y dispositivo de comunicación}

El packaging es un aparato del cual cada bien de consumo tiene que necesariamente dotarse para tener acceso al circuito de las mercancías. Se presenta como una "forma protética" (Bucchetti, 1999, p. 46):3 para el contenido, que necesariamente debe tener un envase para ser distribuido, vendido y finalmente consumido; para el usuario, para quien el envase se convierte en el medio para ponerse en relación con el producto. Es un artefacto complejo, que se puede analizar bajo dos perspectivas: como objeto de uso (prótesis instrumental) y como dispositivo de comunicación (prótesis comunicativa). El envase es, por una parte, una "prótesis instrumental", que hoy en día 
posibilita el desplazamiento del consumo de un producto, tanto en el tiempo como en el espacio (Gros-Pietro, 1995): por un lado, prolonga su vida útil (tiempo) y, por otro, garantiza su transporte desde otros lugares geográficos (espacio). Como prótesis instrumental, el envase es un objeto de uso que se caracteriza por: una dimensión "prestacional", que está relacionada con su naturaleza de "carcasa" o "cáscara" de un contenido, es decir, un contenedor que permite proteger, conservar, transportar, entre otros un producto a lo largo de todo su ciclo de vida, durante las fases posteriores a la producción (venta, consumo y posconsumo); y una dimensión "operativa", que se relaciona con su naturaleza de "herramienta", que facilita la interacción física del usuario con el envase para acceder a su contenido y manipularlo (verterlo, medirlo, aplicarlo, entre otros), principalmente en el contexto de consumo. Asimismo, el packaging se puede también considerar una "prótesis comunicativa", que presenta funciones diversificadas de tipo apelativo, persuasivo, informativo, prescriptivo, entre otros, relacionadas con su naturaleza de "medio de comunicación", "interfaz" o "sistema señalético".

El fortalecimiento de la dimensión comunicativa del packaging supera el concepto de "artefacto" (Anceschi, 1988; Manzini, 1990) -un término que enfatiza a quien lo diseña y lo realiza- y afirma el de "dispositivo de comunicación" (Bucchetti, 1999, pp. 44-47), un término que en cambio incluye el punto de vista de quien se beneficia -anticipando el resultado- de los efectos de la recepción de los mensajes que transmite. La palabra "dispositivo" deriva del verbo "disponer", que implica un sentido de organización estructurada de los elementos, la articulación de las partes, la planificación de un discurso comunicativo (Baule, 2007a; 2007b; Agamben, 2006).

Entre sus funciones comunicativas, el packaging asegura que un producto pueda emerger de la góndola y llamar la atención del potencial comprador; diferenciarse de los productos de la competencia, para asegurar su venta; transmitir los valores de marca y otros significados, para fidelizar al consumidor; informar al usuario sobre la fecha de caducidad de un producto, sus ingredientes y valores nutricionales, a través del etiquetado; explicar el uso tanto del contenedor como de su contenido; proyectar la segunda vida del contenedor, indicando las oportunidades de retornabilidad, reutilización, reciclaje o eliminación, a través del etiquetado medioambiental.

Como enfatiza Valeria Bucchetti (1999, pp. 19-24), la doble naturaleza del envase ha sido considerada, por mucho tiempo, como una "ruptura" $y$ separación de roles y funciones, cuyos orígenes se pueden encontrar en el siglo XVIII cuando, con la primera producción industrial de los envases, se afirmaron dos términos: "embalaje" (desde el francés emballage), para referirse a los aspectos relacionados con el uso y las funciones prestacionales y operativas, vinculadas con las necesidades de contención, protección, apilabilidad, reciclaje, manipulación, entre otros; y "packaging" (desde el inglés to pack), para destacar la dimensión comunicativa, si bien esta última estaba confinada solamente a las funciones decorativas y seductivas del objeto. En otras palabras, el diseño del envase, desde el punto de vista comunicativo, se consideraba exclusivamente como una intervención estilística (styling), es decir una modificación formal sin otro propósito que aumentar el "agrado" por el producto y así aumentar las ventas.

En realidad, la dimensión comunicativa del packaging no se limita únicamente a la expresión de las funciones persuasivas, se extiende a todos los componentes que contribuyen a la activación de los procesos de identificación y 
FUNCIONES DEL PACKAGING

FUNCIONES DE USO

Se refieren al packaging como objeto de uso

(prótesis instrumental)
Función prestacional. Asegura que el contenido del packaging sea conservado, protegido, transportado, almacenado, distribuido y puesto en venta, sin ser dañado o deteriorarse por efecto de las agresiones a las que está sometido el producto a lo largo de su ciclo de vida.

Función operativa. Permite que un usuario -independientemente de sus habilidades, condiciones físicas y psicológicas- interactúe físicamente con el envase, y a través de eso con su contenido, de forma segura y sin desgaste de recursos (tiempo, atención, fuerza, energía, etc.).

Función apelativa. Asegura que el producto, después de llegar al punto de venta, se haga visible y emerja de la góndola, para llamar la atención del comprador potencial y hacerlo acercar al estante. [Funciones relacionadas: función conativa; función señalética; función ostensiva].

Función persuasiva. Hace posible que, en el punto de venta, el producto sea efectivamente elegido y comprado, una vez que haya entrado en contacto con el comprador potencial y que éste último se haya acercado a la góndola. [Funciones relacionadas: función diferenciadora; función estética; función poética; función seductora].

Función identificadora. Alude a la capacidad del packaging de hacerse reconocer, tanto en el punto de venta como en el hogar y otros contextos, de manera más o menos inmediata, y asociar a ciertos destinatarios, a una categoría de producto, a una empresa, etc., y así sucesivamente, con relación a todas sus otras características.

Función expresiva. Permite atribuir al producto un determinado estilo, haciendo alusión a ciertos universos asumidos como referencia y expresando, a través de ellos, valores intangibles de tipo simbólico y afectivo. [Funciones relacionadas: función evocadora; función emotiva].

Función informativa. Se refiere a la naturaleza del packaging como un medio capaz de transmitir mensajes relacionados con el producto o el mismo contenedor, brindando conocimientos, conceptos e información objetiva. [Funciones relacionadas: función cognoscitiva; función referencial].

Función prescriptiva. Orienta al destinatario y le permite ponerse en relación con el contenedor y, a través de ello, con su contenido, transformando el envase en un verdadero "sistema de interfaz"; los mensajes prescriptivos pueden ser explícitos (instrucciones para el uso) o implícitos (affordances).

Función comunicativa extra-producto. Hace referencia al packaging como un mass-medium que permite difundir -además de la información relacionada directamente con el contenedor y su contenido- mensajes de otra naturaleza, destinados a la fidelización del consumidor, la promoción de otros productos o servicios, la difusión de eventos y campañas de sensibilización social.

Función massmediática. Se refiere a la capacidad del packaging de declinar sus competencias comunicativas dentro de otros medios de comunicación (en comerciales televisivos, vallas publicitarias y campañas de prensa, entre otros), cargándose de nuevos roles y funciones; en su condición de "actor massmediático", se vuelve el garante de la emersión del producto en los diferentes contextos.

Tabla 1. Síntesis de las funciones del packaging.

Fuente: Elaboración propia. 
reconocimiento del objeto, las cualidades sensoriales y semánticas, los procesos de fascinación y las formas de acercarse al imaginario del destinatario, así como las distintas formas de interfaz gráfica, la información relacionada con el contenido, las instrucciones de uso, entre otros.

Todos estos aspectos permean el cuerpo entero del objeto, se integran con su funcionamiento y potencian los aspectos prestacionales y operativos: a través de los procesos comunicativos activados por el packaging, se establece con el usuario una "relación dialógica" según la cual cada componente del objeto adquiere valor no tanto "en sí mismo", sino "en relación con" un destinatario. Hasta operaciones muy simples como la apertura de un contenedor y la dosificación de su contenido a veces necesitan "acciones comunicativas" que indiquen al usuario cómo operar, sobre todo cuando no existe un conocimiento previo de una cierta estructura o función; esta necesidad se hace aún más evidente en el caso de soluciones tecnológicamente avanzadas, por ejemplo las "etiquetas inteligentes" (smart labels), cuya presencia dentro del embalaje debe ser comunicada al consumidor para permitir su uso y garantizar, en determinadas circunstancias, una funcionalidad completa.

\section{Funciones del packaging}

Como se ha mencionado, en comparación con la primera producción industrial del siglo XVIII, las funciones del packaging se han multiplicado y han evolucionado notablemente. En origen, el envase era concebido únicamente como un envoltorio para contener y proteger el producto; con el tiempo, se ha seguido asignándole funciones, interrelacionadas e interdependientes, referidas no solamente al contenedor y a su contenido, sino también y sobre todo a la relación con el consumidor (Bucchetti, 1999, pp. 27-29). Muchas de estas funciones corresponden a "responsabilidades" reconocidas originalmente a otros actores, como el mercante o el tendero, que desempeñaban el papel de promotores del producto y garantes de su calidad (Bucchetti, 2005b, p. 14); dicho papel ahora pertenece al packaging, que se ha afirmado como un "vendedor silencioso" (Pilditch, 1973) y el principal protagonista de la distribución de autoservicio (self-service).

El desarrollo de la producción industrial, la afirmación de la gran distribución organizada (GDO) y la expansión de los mercados - hasta incluir a casi todo el planeta, como un mercado global único- han llevado al consumidor a un progresivo distanciamiento desde el producto y su conocimiento, que originalmente se experimentaba, probaba y vivía en contacto directo con el fabricante o comerciante, que eran los únicos intermediarios posibles en el proceso de compra. Este distanciamiento implicó una "transferencia de responsabilidad" al packaging, en la presentación de su contenido: comunicando la información necesaria para identificarlo, conocer sus características, adquirir conocimiento sobre los procesos anteriores a la fase de venta, comprender los modos de uso y consumo, entre otros (Bucchetti, 2005b, pp. 14-21).

El packaging se ha transformado, en ese sentido, en un "objeto mediador". A través del envase se activan formas de "comunicación referencial” $y$-tomando prestado el concepto de la sociología de los procesos culturales y comunicativos (Livolsi, 1983)- de "mediación simbólica"4 de la experiencia inmediata y directa en la transición a la experiencia reflejada y consciente. Los procesos de mediación llevados a cabo por el packaging son particularmente relevantes para al consumidor, justamente por la reducción progresiva de su experiencia directa y la amplificación de su experiencia indirecta, no solo del producto, sino
4. Las diversas formas de expresión que, a través del lenguaje, se configuran como representaciones de la realidad (cuento mitológico, religión, arte, técnicas, conocimiento científico, filosofía, sistemas de derecho, reglas de conducta, entre otros) constituyen las maneras en que el sujeto logra mediar simbólicamente la relación con uno mismo, con los demás, con las cosas. 
5. La expresión "funciones de marketing" hace referencia al papel fundamental que juega el packaging dentro del llamado marketing mix (McCarthy, 1960). también con respecto a su categoría, su empresa o marca, como a los actores y procesos que han determinado su producción, envasado, transporte y venta. De acuerdo con lo anterior, y según Philippe Devismes (1991, p. 20), las funciones del packaging se pueden distinguir en "funciones técnicas", referidas a aspectos tales como la protección y conservación, la compatibilidad con el contenido, la elección de materiales y su especificidad, la apilabilidad, entre otros; y "funciones de marketing",5 referidas, en cambio, a aspectos tales como la apelatividad, la seducción y la persuasión ejercidas sobre el consumidor, la identificación del producto y la información, entre otros.

A partir de la categorización de Devismes (1991), para ampliar la mirada hacia las funciones del packaging con el objetivo de considerar por completo la complejidad del artefacto y favorecer una sistematización orientada principalmente al proyecto, se considera preferible distinguir funciones entre "funciones de uso" y "funciones comunicativas" (ver Tabla 1), refiriéndose respectivamente al packaging como objeto de uso (prótesis instrumental) y como dispositivo de comunicación (prótesis comunicativa).

\subsection{Funciones de uso}

Las funciones de uso, o "funciones instrumentales", del packaging se dividen entre: "función prestacional", cuando se refiere al producto, a su sistema de producción y gestión, a lo largo de todo su ciclo de vida (Mauri, 1996); y "función operativa", cuando se considera la relación del contenedor y del contenido con el usuario.

\subsubsection{Función prestacional}

La función prestacional, o "básica", de un envase asegura que su contenido sea conservado, protegido, transportado, almacenado, distribuido y puesto en venta, sin ser dañado o deteriorarse por efecto de las agresiones a las que está sometido el packaging a lo largo de su ciclo de vida, que pueden ser de tipo físico (golpes, choques térmicos, rayos solares, polvos, entre otros); químico (humedad, agentes de corrosión, contaminantes, entre otros); microbiológico (mohos, esporas, agentes patógenos, entre otros).

La dimensión prestacional del packaging se relaciona con su naturaleza de "contenedor", “carcasa" o "involucro" de un contenido, y considera aspectos tales como su capacidad, volumen, peso, estabilidad, apilabilidad, resistencia, hermeticidad, asepticidad y mantenimiento de las condiciones internas. También concierne otras propiedades más avanzadas, que son propias de los Ilamados "embalajes funcionales" (functional packaging o smart packaging), que implican el uso de un material, un contenedor o un accesorio con una función activa y adicional respecto de las funciones tradicionales de contención y protección, con el objetivo de adaptar el rendimiento del envase a las necesidades y requisitos de un producto o un contexto específico (es el caso, por ejemplo, de los envases aditivados con antimicrobianos y antioxidantes, o de los absorbedores de oxígeno o humedad).

Las prestaciones de un packaging deben respetar criterios de eficacia, eficiencia y economicidad, no solamente en relación con las características peculiares de su contenido, sino también en función de los vínculos determinados por la adopción de específicas tecnologías de envasado, la organización del sistema de distribución, las normativas vigentes, los distintos actores involucrados en su producción y uso, entre otros que influyen directamente en la definición de forma, volumen, estructura, materiales, entre otros del envase. 


\subsubsection{Función operativa}

La función operativa del packaging permite que un usuario -independientemente de sus habilidades, condiciones físicas y psicológicas- interactúe físicamente con el contenedor, y a través de eso con su contenido, de forma segura y sin desgaste de recursos (tiempo, atención, fuerza, energía, entre otros). La dimensión operativa del packaging se refiere a su naturaleza de "herramienta", que facilita la interacción física del usuario con el producto, en el contexto de uso/consumo, para acceder al contenido y manipularlo: se relaciona con los puntos de agarre, los sistemas de apertura y recierre, los mecanismos de dispensación, vertimiento y dosificación del producto; permite al usuario aferrar y transportar el contenedor, acceder a su contenido, separarlo en porciones, utilizarlo y consumirlo en más de una ocasión y en momentos distintos, entre otros.

Es posible interactuar con el envase para reducir su volumen (aplastamiento, separación, doblaje, separación en partes, entre otros) y así acompañar el consumo progresivo del producto o facilitar la eliminación al final de su vida útil. A través de algunas tipologías de packaging, se pueden también llevar a cabo operaciones para transformar el contenido y facilitar su uso y consumo (mezclamiento, enfriamiento, calentamiento, cocción, entre otros), por ejemplo, en el caso de algunos productos que se mezclan en el momento mismo de su erogación (algunos licores, en el ámbito alimentario, o algunos adhesivos bicomponentes usados en la reparación y decoración). La operatividad del envase se refiere a la presencia de "pluses de servicio" ofrecidos al usuario para facilitar la interacción con el contenedor, acceder a su contenido y utilizarlo de manera más eficaz, eficiente y satisfactoria. La dimensión operativa del packaging va más allá de las funciones prestacionales del artefacto, toma en consideración las habilidades del usuario y los objetivos que se quieren lograr a través de la interacción con el envase. El concepto de operatividad en este sentido puede ser asociado a aquello de "usabilidad", un neologismo que no forma parte del diccionario de la Real Academia Española (RAE), aunque es bastante habitual en el ámbito de la informática y la tecnología. La usabilidad se puede definir como "la eficacia, eficiencia y satisfacción con la que un producto permite alcanzar objetivos específicos a usuarios específicos en un contexto de uso específico" (Norma Iso 9241). La usabilidad de un objeto expresa en otras palabras el grado de "facilidad" con que las personas pueden llevar a cabo una interacción, utilizando una herramienta particular u cualquier otro objeto fabricado, con el fin de alcanzar un objetivo concreto. ${ }^{6}$

\subsection{Funciones comunicativas}

Las funciones comunicativas del packaging constituyen un conjunto heterogéneo y complejo -un "haz de funciones" (Anceschi, 1988; Bucchetti, 1999)que se diversifican y toman mayor o menor importancia dependiendo del momento (fase específica del ciclo de vida) o del lugar (contexto de venta, uso y consumo, entre otros) en que el envase se encuentra actuando.

\subsubsection{Función apelativa}

La función apelativa del envase, también llamada "conativa" (del latín conatus, "inicio") de acuerdo con la clasificación de las funciones comunicativas del lenguaje (Jakobson, 1963), asegura que el producto, después de llegar al punto de venta, se haga visible y emerja de la góndola, para llamar la atención del comprador potencial y hacerlo acercar al estante.
6. Sobre la usabilidad de los objetos y la evaluación de los requisitos ergonómicos de los objetos de uso, ver por ejemplo el texto de Francesca Tosi (2005). En relación con la usabilidad de las interfaces web, ver el trabajo de Jakob Nielsen (1999). 
Desde este punto de vista, el packaging se presenta como dispositivo de señalización (función señalética o señalativa), en contextos de venta cada vez más saturados de productos, donde ocurren fenómenos de saturación e inflación, que implican para el consumidor una condición de "híper-selección", es decir multiplicación de tipologías y variedades (Bucchetti, 1999, pp. 36-40). El carácter señalético debe confrontarse con los procesos de "atencionalidad" del destinatario, las oscilaciones entre la atención voluntaria y la atención involuntaria o pasiva (Williams, 1982): debe ser capaz de activar el proceso de puesta en contacto y desencadenar el "enganche visual", es decir, el mecanismo por el cual el envase atrae al destinatario y llama su atención, para permitir la posterior activación de otra forma de diálogo (Bucchetti, 1999, p. 60). La función apelativa se apoya principalmente en el área primaria del packaging (Bucchetti, 1999, p. 101 ss.), que es el área generalmente expuesta en el punto de venta, que incluye elementos de identificación tales como marca, nombre y especificación del producto, representación del contenido, propiedades o características peculiares, eventuales ofertas y promocionales. Esta área no necesariamente coincide por completo con una de las caras geométricamente definidas por la estructura del envase. El lenguaje utilizado es predominantemente icónico y el proceso de comunicación es breve y acelerado. Su función es "hacer ver" (mostrar), junto con "hacer valer" (poner en valor) y "hacer desear" (seducir): en otras palabras, permite exhibir el producto, llamar la atención y el interés, seducir y fomentar la compra, transmitir los valores de la marca, presentar e identificar el producto, la línea, la categoría, entre otros. La "apelatividad" está estrechamente vinculada con acciones comunicativas de tipo ostensivo (Bucchetti, 1999, p. 103): la puesta en exhibición del producto en el punto de venta, asegura que el producto sea visible y emerja desde el estante, para llamar la atención del comprador potencial (función ostensiva).

\subsubsection{Función persuasiva}

El packaging es lo que hace posible que, en el punto de venta, el producto sea efectivamente elegido y comprado, una vez que haya entrado en contacto con el comprador potencial y que este último se haya acercado a la góndola. La función persuasiva se refiere al "rol diferenciador" que asume el envase en el contexto de venta (función diferenciadora) y, por lo tanto, al factor discriminante de la elección de compra (Bucchetti, 1999, pp. 40-44). La dimensión estética, o poética (Jakobson, 1963), juega en este sentido un papel determinante en marcar la diferencia entre productos parecidos. Los recursos, más allá de la calidad gráfica de detalle a nivel configurativo, son variados y pueden incluir, entre otros: las figuras retóricas (por ejemplo, un frasco de miel disfrazado de abeja), las hibridaciones comunicativas (por ejemplo, ciertos envases de espumas de baño que se parecen latas de bebida) y los juegos narrativos (es el caso de la Cajita Feliz de McDonald's graficada con personajes de películas o los envases transformados en juguetes, en forma de toy-packaging). Tal como en la fase de puesta en contacto, también en la fase de elección, el área primaria juega un papel determinante en seducir e incentivar la compra (función seductora), aun cuando es el packaging en su totalidad (al ser agarrado, tocado y examinado) el que finalmente influencie la decisión final del comprador. Se enfrentan, como señala Dina Riccò (2007), dos condiciones de fruición sensorial típicas: una fruición en distancia donde solo se puede ver el producto (por ejemplo, expuesto en una vitrina, o en un estante inaccesible); una fruición sensorial de contacto, que permite manipular el envase y, por lo 
tanto, tener respuestas táctiles, propioceptivas (peso y consistencia), olfativas, incluso gustativas o viscerales en caso de que el contacto se transforme posteriormente en inclusión de un objeto/alimento. "Esta última condición permite la exploración directa de todas las sensaciones, junto con efectos inevitables de interacción, que no son necesariamente congruentes. Por ejemplo, indicios visuales que comunican 'ligero' y verificaciones propioceptivas que, por el contrario, indican 'pesado'” (Riccò, 2007, p. 15).

\subsubsection{Función identificadora}

La identificabilidad del packaging alude a la capacidad de hacerse reconocer -por medio del conjunto de sus propiedades sensoriales y semánticas- tanto en el punto de venta como en el hogar y en otros contextos, de manera más o menos inmediata.

Más allá de las declaraciones explícitas presentes en su área primaria, la función identificadora permite que el producto sea fácilmente reconocido como perteneciente a una cierta categoría (por ejemplo, productos alimenticios o detergentes para la limpieza del hogar); orientado a destinatarios específicos (por ejemplo, productos para niños o adultos mayores); producido por una cierta empresa (es el caso de esos productos cuyos contenedores se han vuelto icónicos de sus marcas, como en el caso de Milka, Toblerone o Nutella); caracterizado por un cierto posicionamiento (por ejemplo, alta gama o primer precio); y así sucesivamente, con relación a todas sus otras características. Junto con el color y otros elementos gráficos, la morfología de un packaging juega un papel comunicativo determinante en los procesos de identificación. No es raro, en la vida cotidiana, que algunos productos -sobre todo los de consumo diario-, sean nombrados solamente a través de su estructura y contenido (por ejemplo, "botella de agua”, "pote de yogur", "tubo de pasta de dientes", "lata de cerveza”, "caja de leche”, entre otros). La referencia es aún más explícita e inmediata cuando está cultural y socialmente establecida y codificada. Las distintas estructuras ${ }^{7}$ de packaging se originaron y afirmaron históricamente, no solamente por intervenciones de tipo formal, sino también por necesidades de tipo funcional, vinculadas con específicos procesos de envasado (es el caso de algunas formas de envases flexibles, como el flow-pack, o de algunas marcas, como Tetra Pak cuyo mismo nombre deriva de su primer envase en polilaminado, con forma de tetraedro, desarrollado en 1946) o con determinados modos de uso y consumo del producto (el cuello estirado y plegado sobre sí mismo de la botella de AnitraWC / PatoPurific, además de recordar a un pato y fortalecer la identidad del producto, permite una aplicación más fácil del detergente).

La dimensión morfológica del envase se refiere a su "componente táxico" (Greimas, 1987; Floch, 1995): ${ }^{8}$ comparando las características distintivas de las estructuras existentes, es posible distinguir familias o subfamilias de envases que se pueden organizar y dividir en categorías (Bucchetti, 1999). Además de las formas estándares, es decir, utilizadas para productos de sectores diferentes (por ejemplo, latas, cajas, frascos, entre otros), se pueden distinguir las formas que identifican categorías de productos (es el caso de las botellas de vino, que están asociadas, de acuerdo con la morfología de cada una, a diferentes variedades de productos: bordelesa, albeisa, champañesa, rhin, fiasco, entre otros) y las formas ad hoc (es el caso, por ejemplo, de los envases de ciertas aguas, como Perrier o Tŷ Nant, o bebidas, como Orangina o Coca-Cola, cuya botella contour ${ }^{9}$ hace referencia inmediata a su
7. Es posible encontrar un compendio de las principales estructuras de packaging en Bucchetti y Ciravegna (2009, pp. 33-70).

8. Parte de los modelos de análisis semiótico de los objetos, incluso el packaging, hacen referencia al modelo descriptivo propuesto por Algirdas Julien Greimas (1987), posteriormente adaptado por Jean Marie Floch (1995) a su análisis del cuchillo Opinel (Floch, 1995: 198-230). Floch (1995) distingue tres niveles diferentes de pertinencia del objeto, que corresponden a tres aspectos en los que se juega su significación: el "componente configurativo", es decir, el momento descriptivo en el que el objeto se descompone en unidades significantes; el "componente táxico", es decir, la comparación de diferentes objetos de la misma tipología, en sus rasgos distintivos; el "componente funcional", es decir, la indagación sobre las acciones posibilitadas por el objeto y el "universo profundo" ("axiológico") involucrado.

9. Botella creada en 1915 y rediseñada por Raymond Lowey en 1954. 
marca, incluso cuando está privada de cualquier signo gráfico (en su versión en blanco, utilizada para testeos).

\subsubsection{Función expresiva}

El packaging, a través de sus componentes comunicativos diseñados, permite atribuir al producto un determinado estilo expresivo, junto con, haciendo alusión a ciertos universos asumidos como referencia y expresando, a través de ellos, valores intangibles de tipo simbólico y afectivo. Mientras que la función identificadora se refiere a la dimensión denotativa del envase, la función expresiva, llamada también evocadora o emotiva (Jakobson, 1963), se relaciona con su dimensión connotativa.

A través de los diferentes acabados superficiales, por ejemplo, es posible atribuir a un soporte propiedades sensoriales -más comúnmente visuales y táctiles- que permiten connotar el envase, e indirectamente su contenido, con valores y significados que van más allá de la dimensión estrictamente denotativa del objeto. Las terminaciones superficiales que actualmente se ocupan para mejorar la apariencia de un soporte, aportándole valor agregado luego de su impresión, se han multiplicado notablemente gracias a los avances tecnológicos de los procesos tanto de impresión como de post-impresión; se pueden mencionar los barnizados (barnices y lacas), los relieves (cuño o sello seco, timbrado, gofrado, entre otros), los laminados (texturizados, metalizados, entre otros), los entelados o los flocados.

Entre los efectos de superficie que se pueden obtener con dichos procesos, además de los más comunes (opaco, brillante, satinado, liso, rugoso, texturizado, altorrelieve, bajorrelieve, entre otros), se puedes también lograr otros más finos y avanzados (nacarado, iridiscente, fosforescente, holográfico, entre otros). El efecto de superficie es una propiedad esencialmente cualitativa, perceptible con la vista y el tacto, difícilmente medible.

El estilo expresivo de los envases es particularmente relevante en la transmisión y fortalecimiento de los valores de marca, pero puede ser de difícil gestión durante el proceso de diseño, debido a que la connotación se refiere a los aspectos metafóricos y simbólicos y puede variar dependiendo del contexto sociocultural, geográfico o de venta (el mismo producto en una tienda monomarca puede implicar evocaciones muy diferentes en comparación con un supermercado).

\subsubsection{Función informativa}

La función informativa -cognoscitiva o referencial (Jakobson, 1963)- del envase se refiere a su naturaleza de medio de comunicación capaz de transmitir mensajes relacionados con el producto o el mismo contenedor, brindando conocimientos, conceptos e información objetiva. Dicha transmisión puede ocurrir en el contexto de la venta, actuando como un componente adicional para diferenciar el producto y guiar la elección del comprador potencial; en el contexto de consumo, como un elemento adicional de conocimiento del producto para su mayor comprensión y mejor fruición para el usuario. Los mensajes varían en función del tipo de producto: por ejemplo, los alimentos, informan sobre su caducidad y durabilidad, los métodos de preservación o la presencia de cualquier sustancia alergénica; los productos que pueden ser dañinos para la salud o que, si se usan de forma incorrecta, pueden representar un peligro, indican precauciones de uso o advertencias específicas, como las relacionadas con la inflamabilidad o la toxicidad. 
Considerando la relevancia y responsabilidad que cada mensaje puede asumir para el destinatario, las funciones informativas se pueden distinguir en: primarias, si están relacionadas con mensajes necesarios para proteger la salud y la seguridad del consumidor (la fecha de caducidad de los alimentos frescos, las advertencias de peligro para productos altamente inflamables o tóxico, entre otros); secundarias, si están orientadas a aumentar el conocimiento y la sensibilización de los consumidores sobre el producto o su envase, destacando propiedades específicas no evidentes (los beneficios del consumo de un alimento para la salud o los efectos del uso de un detergente para la higiene del hogar, así como los impactos positivos en el medio ambiente de un packaging realizados según criterios de eco-diseño); accesorias, si se refieren a información adicional que constituye una profundización o una digresión respecto del producto y que se convierte en una oportunidad para activar discursos comunicativos, relacionados con otros universos de referencia (por ejemplo, recetas, consejos o testimonios atribuidos a expertos $\mathrm{u}$ otros usuarios que ya han utilizado el producto).

La función informativa se apoya principalmente en el área secundaria del packaging (Bucchetti, 1999, p. 103), donde el lenguaje utilizado es predominantemente verbal y el proceso de comunicación se extiende y se ralentiza. Esta área, de hecho, está "vinculada a un momento posterior en el conocimiento del producto" (Bucchetti, 1999, p. 103); su función ya no es "la simulación de la apariencia, la representación del contenido para permitir el reconocimiento y la identificación, sino la descripción, el conocimiento, la prescripción" (Bucchetti, 1999, p. 103), es decir "dar a conocer" (información nutricional, caducidad, composición, entre otros) e "instruir acciones" (instrucciones de uso, modalidad de preservación, indicaciones de reciclaje, entre otros). Además del área secundaria, el área primaria también puede transmitir contenidos informativos: en este caso, la función informativa se combina con la función persuasiva de orientación a la compra.

\subsubsection{Función prescriptiva}

El packaging, a través de su función prescriptiva, orienta al destinatario y le permite ponerse en relación con el contenedor y, a través de ello, con su contenido; de esta forma, el envase se transforma en un sistema de interfaz. La necesidad de proporcionar indicaciones sobre cómo interactuar con el envase o el producto está vinculada con la necesidad de acercar al consumidor a objetos que, respecto al pasado, ya no tienen una fuerte identidad técnico-cultural (entre las causas: la creación de nuevos productos y envases, las formas de hibridación que ocurren entre categorías de productos y tipologías de contenedores, tanto a nivel de materiales y formas, como en los significados simbólicos y culturales); además, son cada vez más complejos por el aumento continuo de pluses de tipo prestacional y operativo (nuevos sistemas de apertura, erogación, conservación, entre otros), que no siempre son claramente comprensibles (Bucchetti, 1999, pp. 61-64). Los mensajes prescriptivos se pueden dividir entre "instrucciones explícitas", es decir, formas de ejemplificaciones gráficas que explican las "disposiciones" para impartir, a través de un guion gráfico (storyboard) constituido por una secuencia de palabras, elementos textuales y/o icónicos (pictogramas, ilustraciones, fotografías) que describen las distintas acciones (Candusso y CaravieIlo, 1994; Mijksenaar y Westendorp, 1999); e "instrucciones implícitas", es decir, "invitaciones" a llevar a cabo acciones en base a los affordances (Gibson 1979; 
10. El concepto de "factitividad" está en la base de la reflexión de Michela Deni (2002), que indaga en particular las "potencialidades de los objetos, como procesos de significación, para influir y prescribir acciones y prácticas de uso" (Deni 2002, p. 11) y destaca como "Ios mismos objetos, al ser elementos significantes, inducen comportamientos individuales y sociales y por ende modifican las relaciones intersubjetivas" (Deni, 2002, p. 11)

11. Michela Deni (2002) define el "usuario modeIo" refiriéndose al "lector modelo" de Umberto Eco (1979; 1994) como "el usuario tipo que el objeto no solo proporciona como colaborador, sino que también intenta crear. Al contrario, el usuario empírico es quien usa realmente el objeto y puede hacerlo para fines que, aparentemente o no, no tienen nada que ver con el objeto mismo" (Deni, 2002, p. 11).
Norman 1988, 1998) inscritos en la morfología o en los acabados superficiales del envase, que guían y ayudan a usar correctamente cada parte del objeto. Affordance es la cualidad de un objeto o ambiente que permite -o autoriza, desde el verbo inglés to afford- a un individuo a realizar una acción (la forma redonda y la superficie moleteada de la tapa de una botella de plástico permite, por ejemplo, la acción de desenroscar; un mango moldeado según la forma de la mano permite un agarre, entre otros): el packaging, en cuanto destinado al objeto, presenta elementos que actúan a nivel del hacer y son interpretados por los usuarios como pistas y como indicadores de las posibles acciones que se pueden lograr sobre ello y a través de ello. Los affordances se pueden poner en relación con la "dimensión factitiva" (Deni, 2002) del packaging: ${ }^{10}$ los objetos cotidianos, incluso el envase, están dotados de intencionalidad fenomenológica al incluir dentro de sí mismos una cierta manera de entregarse a un sujeto y una cierta forma de relacionarse con el mundo, encarnando un conjunto de escenarios de uso (los llamados programas narrativos) y construyendo a su propio usuario modelo. ${ }^{11}$ En el proyecto de la dimensión prescriptiva, es importante considerar aspectos como la modalidad de representación (elementos verbales e icónicos, la dimensión morfológica, los acabados superficiales, entre otros); el tipo de contenido que se transmite (qué hacer o evitar, dónde y cómo hacerlo, con cuáles accesorios, entre otros); el número y la complejidad de las acciones que se necesita llevar a cabo (inmediato/simple vs. complejo); la localización de las instrucciones o affordances respecto al punto de manipulación (localizados vs. deslocalizados); la reducción o eliminación de affordances falsos, es decir, no esperados durante el proceso de diseño y que pueden constituir un engaño para el usuario (por ejemplo, una falsa indicación de apertura). Los contenidos prescriptivos, de manera similar a los de tipo informativo, se colocan en particular en el área secundaria, donde el lenguaje utilizado es predominantemente verbal y el proceso de comunicación se extiende y se ralentiza; sin embargo, se pueden también encontrar en el área primaria, donde se combinan y fortalecen la orientación persuasiva hacia la compra.

\subsubsection{Función comunicativa extra-producto}

Desde la función primaria de "garante" de la calidad del producto, a lo largo del tiempo el packaging ha incrementado sus funciones comunicativas y se ha transformado en un medio adecuado para trasmitir contenidos comunicativos de diverso tipo, llegando a actuar como un verdadero mass-medium que permite difundir, no solamente la información relacionada directamente con el contenedor y su contenido (función informativa y prescriptiva), sino también mensajes de otra naturaleza, o "extra-producto". Dichos mensajes se han "estratificado", también a través de formas de hibridación comunicativa, en la superficie del envase (Ciravegna, 2007; Diana, 2006) y contribuyen a la formación del gusto, la construcción del imaginario, y a la orientación de los comportamientos del destinatario.

Entre los contenidos comunicativos extra-producto se encuentran los mensajes destinados a la fidelización del consumidor, a través de juegos, concursos y recolección de puntos, así como la promoción de otros productos o servicios, sobre la base de acuerdos de co-marketing o partnerships comunicativos con otras empresas (por ejemplo, para el lanzamiento de una película o un videojuego). Junto a estas iniciativas más marcadamente comerciales, se pueden encontrar también la difusión y promoción de eventos culturales 
(exposiciones de arte, conciertos y festivales), eventos deportivos (campeonatos, olimpiadas, entre otros) y campañas de sensibilización social sobre temas de utilidad pública (el ejemplo más significativo es probablemente el missing presente en los cartones de leche de los años setenta en los Estados Unidos, para apoyar la búsqueda de niños desaparecidos). ${ }^{12}$

El packaging se propone en este sentido como un soporte sobre el que se articula una narración compleja alrededor del producto: si la dimensión estrictamente informativa satisface una necesidad racional de conocimiento del producto -como resultado de la necesidad práctica de ponerse en contacto con un contenido ocultado por el envase-, en cambio, la dimensión narrativa satisface una necesidad más bien emocional, de seducción y fascinación (Bucchetti, 1999).

\subsubsection{Función massmediática}

Por último, la función massmediática se refiere a la capacidad del envase de declinar sus competencias comunicativas dentro de otros medios de comunicación (en comerciales televisivos, vallas publicitarias y campañas de prensa, entre otros), cargándose así de nuevos roles y funciones. En comparación con la función comunicativa extra-producto, se distingue, de hecho, "el involucro narrador, que se propone como un medio de comunicación, es decir, el envase que se encuentra en [...] la grande distribución, y las expresiones que asumirá en cambio una vez que será narrado a través de los otros medios de comunicación" (Bucchetti, 1999, p. 55).

El packaging, en su condición de "actor massmediático", se vuelve el garante de la emersión del producto en los diferentes contextos, gracias por ejemplo al pack-shot, es decir, la imagen del envase del producto aislada y puesta como firma de un anuncio, fungiendo como enganche, enlace y continuidad entre los lugares virtuales de la publicidad y los lugares reales de venta y consumo (Bucchetti, 1999, pp. 54-57).

A diferencia de lo que sucede en otros ámbitos - como por ejemplo en el punto de venta, donde la comunicación también pasa por otras modalidades sensoriales - la "fruición" del envase en el espacio publicitario es casi exclusivamente visual. ${ }^{13}$ Esto implica que el diseño del envase tenga en cuenta su "visibilidad" y ponga atención sobre cómo se cuenta el producto y cómo se reproduce dentro de un comercial o afiche. Por lo tanto, el packaging debe tener atributos de lo que Giovanni Anceschi llama en italiano icogenia (Anceschi 1992C, p. 183), es decir, una predisposición a la figurabilidad y representación (fotográfica, televisiva, web): el envase debe poder "someterse a deformaciones, a nuevas enfatizaciones de perspectiva, pero sobre todo debe responder a ley de la pregnancia y poseer rasgos que garantizan la reconocibilidad y produzcan una identificación inmediata" (Bucchetti, 1999, p. 57).

\section{Sistema-packaging}

La panorámica sobre las distintas funciones del packaging permite tener una visión general y entender la complejidad y las cualidades multifacéticas de este artefacto, que a la vez se presenta como un objeto de uso con funciones de tipo instrumentales y operativos, y un dispositivo de comunicación con carácter apelativo, persuasivo, informativo, prescriptivo, entre otros. El envase presenta una naturaleza multifacética que implica diferentes habilidades y, por lo tanto, involucra múltiples disciplinas.

Al mover la atención del packaging como artefacto a su proyecto, es decir, a todas las actividades de investigación, ideación y desarrollo orientadas a su
12. Cabe señalar, sin embargo, que incluso en el caso de los ejemplos más virtuosos (como las campañas sociales de utilidad pública), las acciones comunicativas antes descritas responden a lógicas de tipo comercial, ya que finalmente contribuyen a aumentar las ventas de los productos y, utilizando una expresión de Guy Debord (1967), a la "espectacularización generalizada" de los bienes: de acuerdo con Jean Baudrillard (1968), al "nihilismo de consumo" se contrapone una especie de "humanismo de consumo", es decir, se ofrecen a las personas la aprobación y la justificación para disfrutar de los productos que compran, para mostrar que hacer la vida placentera con ellos no es inmoral sino moral.

13. Se han dado casos de publicidad multisensorial, que han visto la estimulación de otros sentidos, por ejemplo, el olfato, pero sin llegar a resultados particularmente convincentes en términos de efectividad comunicativa. 
14. Ver la sección "Packaging” (editado por L. Badalucco, V. Bucchetti, F. Jégou, S. Maschi) del proyecto de investigación “Il ruolo del Disegno Industriale per l'innovazione di prodotto. Sviluppo delle risorse progettuali del Sistema Italia tra risorse locali e mercati globali [El rol del Diseño Industrial para la innovación del producto.

Desarrollo de los recursos proyectuales del Sistema Italia entre recursos locales y mercados globales]" (1998-2000), responsable científico: E. Manzini; coordinación: D. Bruno, C. Conio, S. Maffei, D. Riccò, D. Sangiorgi, G. Simonelli, F. Zurlo (Departamento de Diseño Industrial y Tecnología de la Arquitectura, Politecnico di Milano). El proyecto de investigación, cofinanciado por el ministerio italiano de la universidad e investigación (MIUR - Ministero dell'Università e della Ricerca), sentó las bases para lo que luego se convirtió en "SDI | Sistema Design Italia".

15. La expresión "sistema-packaging" y su definición hacen referencia al llamado "sistema-producto" definido en Mauri (1996). realización, es posible reconocer, dentro del marco general de las disciplinas del Diseño, un área -el diseño de packaging, precisamente- que se coloca en el cruce entre distintas disciplinas (diseño de comunicación, diseño de producto, diseño de interacción, diseño para la sostenibilidad, diseño estratégico, entre otros) y está en una fuerte relación dialéctica con otras áreas del conocimiento (semiótica, marketing, ergonomía, ciencia y tecnología de los materiales, entre otros).

Se trata de una especie de área fronteriza que solamente en las últimas décadas ha comenzado a definir su propia identidad autónoma, junto con un núcleo específico de conocimientos y competencias (Bucchetti, 2005a; 2010). Las razones de este retraso se encuentran, en primer lugar, precisamente en la dimensión multidisciplinar del proyecto de packaging, que se caracteriza por una multiplicidad y heterogeneidad de actores involucrados que intervienen en las variadas etapas del proceso, cada uno con un rol distinto en el proyecto del envase.

De acuerdo con los resultados de una investigación realizada dentro del sector del packaging en Italia, en el contexto del Ilamado Sistema Design Italia, ${ }^{14}$ el diseño de un envase se presenta como una cadena proyectual que, como la económica, está fragmentada en el tiempo y el espacio. En otras palabras, el proceso de diseño se genera mediante una serie de intervenciones sucesivas e incrementales que tienen tiempos, modos y propósitos diferentes. El proyecto de un envase, por lo tanto, no es atribuible a un único actor del sistema y debe entenderse como el resultado de un conjunto integrado de elecciones realizadas por una pluralidad de actores que desempeñan, cada uno, un papel específico -directo o indirecto- en la definición del packaging. Dichos actores, junto con las relaciones (por ejemplo, intercambios de información) que entre ellos se establecen para normarlo, diseñarlo, producirlo y gestionarlo a lo largo de todo su ciclo de vida, conforman el llamado "sistema -packaging" ${ }^{\prime 15}$ (Ciravegna, 2010) y se pueden agrupar en las siguientes áreas:

- Área del proyecto y de la gestión estratégica: estudios de diseño gráfico, agencias de branding, estudios de diseño industrial, prototipado e ingenierización, entre otros.

- Área de la producción: productores de materias primas y materiales de embalaje, fabricantes y transformadores (convertidores) de envases, fabricantes de máquinas para el envasado, entre otros.

- Área de las empresas usuarias: empresas multinacionales (o trasnacionales), grandes empresas, pequeñas y medianas empresas (Pymes), microempresas, empresas familiares e individuales, entre otros.

- Área de la distribución: minoristas (venta al detalle), mayoristas (venta por mayor), almacenes, supermercados e hipermercados (Gran Distribución Organizada, GDO), operadores logísticos y del transporte, importadores y exportadores, entre otros.

- Área de los usuarios finales: consumidores finales individuales y comunidades, cooperativas, hospitales, Ho.Re.Ca (hoteles-restaurantes-catering), entre otros.

- Área de la prevención y gestión del final de vida: gestores y operadores de la recolección de los desechos, de los rellenos sanitarios y vertederos, de los procesos de reciclaje, de la recuperación energética, entre otros.

- Área de la legislación y normalización técnica: ministerios y otras administraciones públicas, organismos de supervisión y control, institutos 
de normalización, así como otros organismos gubernamentales y no gubernamentales; entre otros.

- Área de la investigación y experimentación: laboratorios de análisis y certificación, centros de I+D+i (investigación, desarrollo e innovación) públicos y privados, universitarios, corporativos, entre otros.

- Área de la difusión y promoción: revistas, observatorios, museos y colecciones, gremios y asociaciones comerciales, consorcios, entre otros.

- Área de la educación y formación: universidades, institutos técnicos, centros de formación profesional y educación continua, entre otros.

\section{Conclusiones: complejidad del proyecto y rol mediador del diseño}

Las consecuencias del aumento progresivo de complejidad del sistemapackaging y la fragmentación de los procesos de definición y producción de un envase, causada por la multiplicidad y heterogeneidad de actores involucrados, se hacen aún más evidentes si se considera la dificultad de diálogo entre el mundo tecnológico de la producción (las oficinas técnicas de las empresas, los centros de $1+D+i$, entre otros que se ocupan de las prestaciones y el "funcionamiento" del envase) y el área "creativa" del proyecto (las agencias, los estudios de diseño o los diseñadores individuales, que se centran principalmente en la definición de la "forma" del envase), cuya relación se complica también, en la mayoría de los casos, cuando no es directa y está mediada por las empresas usuarias.

Esta oposición, sin embargo, se puede superar gracias a una figura renovada del diseñador que, al mantener una visión general de todo el sistema-packaging, sea capaz de crear un "puente" entre los diferentes actores involucrados, favoreciendo una "convergencia" de los diferentes puntos de vista y necesidades,

así como la integración de las funciones del artefacto, conectando la dimensión comunicativa con los aspectos más estrictamente prestacionales y operativos (Bucchetti 2005a, p. 42).

El perfil de diseñador que se delinea, se ve reflejado en la definición de Diseño formulada por Tomás Maldonado (1991) que enfatiza justamente el enfoque sistémico y las habilidades de síntesis del diseñador, cuya actividad consistiría en coordinar, integrar y articular todos aquellos factores que, de una manera $u$ otra, participan en el proceso constitutivo de la forma del producto. $Y$, más precisamente, se alude a los factores relacionados con el uso, la fruición y el consumo individual o social del producto (factores funcionales, simbólicos o culturales), así como a los que están relacionados con su producción (factores técnico-económicos, técnico-constructivos, técnico-sistémicos, técnico-productivos, técnico-distributivos) (Maldonado 1991, p. 12).

El Diseño asume, por lo tanto, un papel de "dirección" y "mediación" (Celaschi 2008), capaz de dar forma, a través de la "síntesis proyectual", a soluciones de packaging que sean una expresión de la confluencia de los requerimientos y vínculos expresados por las distintas partes implicadas, así como por la naturaleza multifacética del artefacto.

Desde esta perspectiva, la sistematización de un marco conceptual para el área del diseño de packaging, permite enfrentar la complejidad del proyecto: por un lado, comprendiendo y gestionando las relaciones que se establecen entre los actores y ámbitos disciplinares involucrados en su desarrollo, producción, venta, consumo, entre otros; y, por otro lado, controlando e integrando las múltiples funciones del envase. 


\section{Referencias}

Agamben, G. (2006). Che cos'è un dispositivo [¿Qué es un dispositivo?]. Roma: Nottetempo.

Anceschi, G. (1988). Monogrammi e figure [Monogramas y figuras]. ( $2^{a}$ ed.). Firenze: La Casa Husher.

Anceschi, G. (1992a). II pensiero protetico [El pensamiento protético]. Ottagono. (102), 9-13.

Anceschi, G. (1992b). L'involucro transitorio [El envoltorio transitorio]. Imballaggio. (439), 125.

Anceschi, G. (1992C). L'oggetto della raffigurazione [El objeto de la representación]. Milano: Etaslibri.

Baudrillard, J. (1968). Le système des objets [El sistema de los objetos]. Paris: Édition Gallimard.

Baule, G. (2007a). Dispositivi di comunicazione [Dispositivos de comunicación]. LineaGrafica. (367), 12-13.

Baule, G. (2007b). Lessico [Léxico]. En V. Bucchetti. (Ed.), Culture visive [Culturas visuales] (pp. 13-57). Milano, Italia: Edizioni POLI.design.

Bucchetti, V. (1999). La messa in scena del prodotto [La puesta en escena del producto] ( $1^{\text {a }}$ ed.). Milano: FrancoAngeli.

Bucchetti, V. (2005a). Discipline del packaging design [Disciplinas del diseño de packaging]. LineaGrafica. (353), 38-49.

Bucchetti, V. (2005b). Packaging design. Storia, linguaggi, progetto [Diseño de envases. Historia, lenguajes, proyecto]. Milano: FrancoAngeli.

Bucchetti, V. (2010). Packaging design: percorsi disciplinari [Diseño de envases: caminos disciplinarios]. En E. Ciravegna. La qualità del packaging [La calidad del envase] (pp. 11-19). Milano: FrancoAngeli.

Bucchetti, V. y Ciravegna, E. (2009). Le parole del packaging [Las palabras del envase]. Milano: Edizioni Dativo.

Candusso, G. y Caraviello, A. (1994). Le istruzioni per l'uso degli imballaggi dei prodotti di largo consumo. Analisi del codice grafico [Instrucciones para el uso de los envases de los productos de consumo. Análisis del código gráfico]. Tesis de Magíster del Politecnico di Milano.

Celaschi, F. (2008). Il design come mediatore tra bisogni [El Diseño como un mediador entre las necesidades]. En C. Germak (Ed.), L'uomo al centro del progetto [El hombre en el centro del proyecto] (pp. 19-31). Torino: Allemandi.

Ciravegna, E. (2007). Packaging, un nuovo medium? [¿Envase, un nuevo medio?]. LineaGrafica. (367), 60-65.

Ciravegna, E. (2010). La qualità del packaging [La calidad del envase]. Milano: FrancoAngeli.

Debord, G. (1967). La société du spectacle [La sociedad del espectáculo]. Paris: Buchet-Chastel.

Deni, M. (2002). Oggetti in azione [Objetos en acción]. Milano: FrancoAngeli.

Devismes, P. (1991). Packaging. Mode d'emploi [Packaging. Manual de uso]. Paris: Dunod.
Diana, P. (2006). Dalla comunicazione sul prodotto al messaggio sociale. Il packaging come nuovo medium [De la comunicación sobre el producto al mensaje social. El envase como un nuevo medio]. Tesis de Magíster del Politecnico di Milano.

Eco, U. (1973). Il segno [Signo]. Milano: Isedi.

Eco, U. (1975a). Chi ha paura del cannocchiale? [¿Quién le teme al telescopio?]. Op. cit.. (32). Recuperado de http:// www.opcit.it/cms/?p=85

Eco, U. (1975b). Trattato di semiotica generale [Tratado de semiótica general]. Milano: Bompiani.

Eco, U. (1979). Lector in fabula [Lector in fabula]. Milano: Bompiani.

Eco, U. (1994). Sei passeggiate nei boschi narrativi [Seis paseos por los bosques narrativos]. Milano: Bompiani.

Eco, U. (1997). Kant e l'ornitorinco [Kant y el ornitorrinco]. Milano: Bompiani.

Floch, J.-M. (1995). Identités Visuelles [Identidades visuales]. Paris: PUF.

Gibson, J. J. (1979). The Ecological Approach to Visual Perception [El enfoque ecológico a la percepción visual]. Boston: Houghton Mifflin.

Greimas, A. J. (1987). De l'imperfection [De la imperfección]. Paris: Éditions Pierre Fanlac.

Gros-Pietro, G. M. (Ed.) (1995). Libro bianco sull'imballaggio [Libro blanco sobre el embalaje]. Milano: Istituto Italiano Imballaggio.

Jakobson, R. (1963). Essais de linguistique genérale [Ensayos de lingüística general]. Paris: Minuit.

Livolsi, M. (Ed.) (1983). Sociologia dei processi culturali [Sociología de los procesos culturales]. Milano: FrancoAngeli.

Maldonado, T. (1992). Reale e virtuale [Lo real y lo virtual]. Milano: Feltrinelli.

Maldonado, T. (1991). Disegno Industriale. Un riesame [El diseño industrial reconsiderado]. ( $2^{\mathrm{a}}$ ed.). Milano: Feltrinelli.

Manzini, E. (1990). Artefatti. Verso una nuova ecologia deIl'ambiente artificiale [Artefactos. Hacia una nueva ecología del ambiente artificial]. Milano: Domus Academy.

Marsciani, F. y Zinna, A. (1991). Elementi di semiotica generativa [Elementos de la semiótica generativa]. Bologna: Esculapio.

Mauri, F. (1996). Progettare progettando strategia. Il design del sistema prodotto [Proyectar proyectando estrategia. El diseño del sistema de producto]. Milano: Dunod.

McCarthy, E. J. (1960). Basic Marketing. A Managerial Approach [Comercialización: un enfoque gerencial]. Homewood: Richard D. Irwin.

Mijksenaar, P. y Westendorp, P. (1999). Open Here. The Art of Instructional Design [Abrir aquí. El arte del diseño de instrucciones]. New York: Joost Elffers Books. 
Nielsen, J. (1999). Designing Web Usability [Usabilidad, diseño de sitios web]. Indianapolis: New Riders.

Norma ISO 9241 (2006). Ergonomics of Human System Interaction [Ergonomía de la interacción entre humano y sistema]. International Standard Organization.

Norman, D. A. (1988). The Psychology of Everyday Things [La psicología de los objetos cotidianos]. ( $\mathrm{1}^{\mathrm{a}}$ ed.). New York: Basic Books.

Norman, D. A. (1998). The Invisible Computer [El ordenador invisible]. Cambridge: The MIT Press.

Pilditch, J. (1973). The Silent Salesman [El vendedor silencioso]. ( $2^{\mathrm{a}}$ ed.). London: Business Publications.

Riccò, D. (2007). Congruenze sinestesiche [Congruencias sinestésicas]. En V. Bucchetti (Ed.), Packaging tra vista e tatto [El envase, entre la vista y el tacto] (pp. 15-17). Milano: Edizioni POLI.design.

Tosi, F. (2005). Ergonomia, progetto, prodotto [Ergonomía, proyecto, producto]. Milano: FrancoAngeli.

Williams, K. C. (1982). Behavioural Aspects of Marketing [Aspectos comportamentales de la mercadotecnia]. London: Heinemann. 